Revista de Psicología y Educación / Journal of Psychology and Education, 2021, 16(2), 140-160 (www.rpye.es) Doi: https://doi.org/10.23923/rpye2021.02.207

ISSN: $1699-9517$

\title{
Estudio exploratorio observacional de la enseñanza de la composición textual en las aulas
}

\author{
Rut Sánchez-Riverol* ${ }^{*}$, Rui A. Alves² y Raquel Fidalgo' \\ Universidad de León \\ ${ }^{2}$ Universidad de Oporto
}

\begin{abstract}
Resumen: En el presente estudio se aborda, mediante la observación, el análisis del proceso de enseñanza seguido por 7 docentes de $4^{\circ}$ de Educación Primaria para enseñar al alumnado la composición de textos escritos, incluyendo el análisis de qué procesos y conocimientos implicados en la composición textual se enseñan y cómo se enseñan, a través del análisis del proceso instruccional seguido por el profesorado en la enseñanza, así como de los agrupamientos y los materiales empleados. Se realizó un registro audiovisual de 12 sesiones en las que el profesorado enseñó la composición de una tipología textual concreta. Se diseñó un sistema de categorías ad-hoc incluyendo ambas dimensiones de análisis. Los resultados obtenidos mostraron un mayor énfasis en la enseñanza del proceso de redacción ligado a las características específicas de la tipología textual, y un menor énfasis en el proceso de planificación, mientras que el proceso de revisión no fue enseñado explícitamente. Asimismo, el procedimiento instruccional más empleado fue la enseñanza directa, si bien esta se combinó, principalmente, con la ejemplificación y con el análisis de modelos textuales. Se sugiere promover programas de desarrollo profesional en torno a la enseñanza de la composición textual desde un enfoque estratégico y autorregulado.
\end{abstract}

Palabras clave: Enseñanza; Escritura; Observación; Práctica pedagógica.

\section{Exploratory observational study of the teaching of textual composition in classrooms}

\begin{abstract}
In the present study, we analyse, through observation, the instruction process of seven 4thgrade teachers when teaching text compositionto their students. This includes the analysis of which processes and knowledge involved in text composition are taught and how they are taught, including the analysis of instructional procedures, groupings and materials. We conducted audio-visual recordings of 12 sessions in which teachers taught a specific type of writing composition. We designed an ad-hoc system of categories, which included both dimensions of analysis. Results suggest a major emphasis on the drafting process linked to the specific features of each text genreand less emphasis on the planning process, whereas the revision process was not explicitly taught. Besides, teachers mostly used direct teaching, although theycombinedit with exemplification and the analysis of textual models. We emphasize the need to provide teachers with professional development programs on the teaching of text composition based on a strategic and self-regulated approach.
\end{abstract}

Key words: Teaching; Writing; Observation; Pedagogical practice.

Un alto número de estudiantes, tanto a nivel internacional (National Center for Education Statistics, 2003; 2012) como en España (Ministerio de Educación, 2010; 2011) presenta un bajo rendimiento en la escritura, a pesar de tener una actitud positiva hacia su

Recibido: 10/03/2021 - Aceptado: 27/05/2021 - Avance online: 07/06/2021

*Correspondencia: Rut Sánchez-Rivero

Universidad de León. León, España.

Dirección: 24071, León, España.

E-mail: rsanr@unileon.es aprendizaje (García y Fidalgo, 2003; Graham et al., 2007; Gutiérrez, 2013). Concretamente en Estados Unidos, sólo entorno a un 24-27\% del alumnado muestra un nivel de rendimiento adecuado en la escritura (NCES, 2012). En España, en los últimos informes emitidos por el Ministerio de Educación, en los que se evalúa el nivel de rendimiento del alumnado en la competencia lingüística, dentro de la cual se incluye la expresión escrita o habilidad 
de composición textual, se obtiene que sólo un $8 \%$ del alumnado tanto de $4^{\circ}$ de Primaria (Ministerio de Educación, 2010) como de $2^{\circ}$ de ESO (Ministerio de Educación, 2011) muestra un nivel de rendimiento alto en la competencia lingüística. En relación a la composición textual, estos datos indican que un $92 \%$ del alumnado de nuestro país no es capaz de elaborar un texto organizado, que mantenga una progresión temática y que cumpla criterios adecuados de cohesión léxica y gramatical, así como de legibilidad y presentación.

Este bajo rendimiento mostrado por el alumnado denota que, como reflejan diferentes modelos teóricos, la composición escrita es una conducta sumamente compleja en la que intervienen múltiples procesos cognitivos, tanto de bajo como de alto nivel cognitivo (Berninger y Winn, 2006; Hayes, 1996; Hayes y Flower, 1980). Los procesos de bajo nivel cognitivo de la escritura, como la caligrafía, la ortografía y la gramática, se relacionan con la producción o transcripción textual y su dominio demanda una automatización en su aprendizaje. Por su parte, los procesos de alto nivel cognitivo de la escritura, requieren para su dominio una autorregulación y un control consciente de los mismos. Dentro de estos últimos, se incluyen los procesos de planificación, redacción y revisión textual, establecidos por primera vez en el modelo cognitivo de Hayes y Flower (1980) y que se siguen reconociendo como procesos principales en modelos desarrollados con posterioridad (Berninger y Winn, 2006; Hayes, 1996). La planificación implica generar ideas y organizarlas en torno a una estructura textual siguiendo un plan establecido. La redacción supone transformar dichas ideas en un texto escrito atendiendo tanto a aspectos ortográficos, caligráficos y gramaticales, como a aspectos de estructuración y elaboración del texto y su ajuste al lector. Finalmente, la revisión implica la identificación de errores en el texto mediante su lectura y evaluación de acuerdo al plan establecido y la edición del mismo mediante las correcciones necesarias. A su vez, todos estos procesos cognitivos se organizan y coordinan de modo interactivo y recursivo a lo largo de la escritura del texto, lo que hace que el dominio de la composición textual por parte del alumnado sea una tarea difícil y que demande una enseñanza sistemática y planificada, cuya responsabilidad recae de manera directa en la escuela (Alvarado y Silvestri, 2003).

Con el fin de proporcionar al alumnado una enseñanza efectiva que favorezca su rendimiento escritor, desde el ámbito científico han surgido en los últimos años múltiples meta-análisis dirigidos a identificar aquellas prácticas instruccionales, cuya eficacia para la mejora de la composición escrita se encuentra avalada a nivel empírico (ver estudio de Graham y Harris, 2018, en el que se meta-analizan más de 20 meta-análisis). En concreto, en el meta-análisis de Koster et al. (2015) en el que se meta-analizan exclusivamente prácticas instruccionales implementadas en los cursos de $4^{\circ}$ a $6^{\circ}$ de Educación Primaria, se destacaron prácticas instruccionales muy efectivas, con un tamaño del efecto igual o superior a 0.7, como proporcionar al alumnado una instrucción estratégica y autorregulada en los procesos de planificación y revisión textual; el establecimiento de objetivos a considerar en la escritura del texto; la enseñanza explícita de la estructura textual de diferentes tipologías; y proporcionar feedback al alumnado acerca de sus textos. A su vez, con un tamaño del efecto entre 0.4 y 0.6 se destacaron dos prácticas moderadamente efectivas, como la práctica colaborativa en torno a la escritura del texto y la autoevaluación del texto a partir de criterios específicos. Asimismo, también se destaca la observación y emulación de modelos textuales ejemplares y la enseñanza de la escritura a través de las tecnologías que, si bien no se incluyeron en el estudio de Koster et al. (2015), son prácticas que presentan un efecto positivo moderado en el estudio de Graham y Harris (2018).

Conscientes del avance del conocimiento científico en el ámbito instruccional de la escritura, cabe preguntarse por la transferencia real de dicho conocimiento al ámbito educativo y a la práctica docente del profesorado. En esta línea, cobra especial relevancia el 
análisis, a través de la observación, de la práctica implementada por el profesorado para la enseñanza de la escritura. Dicho análisis favorecerá la identificación de posibles lagunas y necesidades de mejora en la práctica implementada, así como el diseño de programas de desarrollo profesional del profesorado ajustado a las mismas (Ciullo et al., 2016). Todo ello con el fin de optimizar la adquisición del dominio de la escritura por parte del alumnado y la respuesta preventiva o de intervención ante posibles dificultades en su aprendizaje (Arrimada et al., 2018; 2019).

Desde nuestro conocimiento, a nivel internacional cabe señalar dos estudios que han realizado una observación de la práctica docente del profesorado de Educación Primaria en torno a la enseñanza de la escritura. En el primero de ellos, Coker et al. (2016) realizaron cuatro sesiones de observación participante de un día escolar en 50 aulas de $1^{\circ}$ de Primaria, con el fin de analizar qué sucedía en relación a la escritura. El análisis incluyó el tiempo dedicado a la enseñanza de diferentes contenidos de escritura y al uso de diferentes procedimientos instruccionales, agrupamientos, materiales y actividades del alumnado. Sus resultados mostraron que, en general, del total del tiempo académico observado durante un día escolar (275 minutos) se dedicaron 26,4 minutos a la enseñanza de la escritura $(9,6 \%)$ y 125 minutos a la escritura por parte del alumnado (46,5\%). Del tiempo dedicado a la escritura por parte del alumnado, la mayoría fue a nivel de palabra mediante actividades que requerían copiar la respuesta o completar con una respuesta correcta, dedicando un menor tiempo a la escritura del alumnado a nivel de composición textual. En relación a la enseñanza de la escritura, el contenido instruccional al que se le dedicó un mayor tiempo fue la enseñanza y/o práctica de las habilidades básicas de la escritura y en especial a la ortografía, seguido de la enseñanza y/o práctica de los procesos de escritura. También, la mayoría del tiempo se trabajó a nivel de grupo-aula y los procedimientos instruccionales más empleados por el profesorado fueron la explicación por parte del profesor sin ningún tipo de respuesta del alumnado o mediante la realización de preguntas-respuestas. En el análisis de dichos resultados es necesario tener en cuenta que la observación de la enseñanza de la escritura se llevó a cabo exclusivamente en primero de Primaria. Por ello, sus conclusiones difícilmente pueden ser generalizables a la enseñanza de la escritura en cursos superiores de la Educación Primaria, donde se asume la automatización de las habilidades básicas de la escritura y un mayor énfasis, por tanto, a la enseñanza de habilidades más complejas como la planificación y la revisión textual (Graham et al., 2012). En este sentido, el estudio observacional de Rietdijk et al. (2018) se desarrolló con 58 profesores de $4^{\circ}$ a $6^{\circ}$ de Primaria. En concreto, realizaron una observación participante y un registro en audio de dos sesiones de enseñanza de la escritura, impartidas de forma ordinaria por el profesorado dentro del horario dedicado a la asignatura de lengua. Su objetivo era analizar el tipo de enfoque que seguía el profesorado para enseñar la escritura de textos, así como el tiempo dedicado a diferentes actividades del aula (instrucción, modelado, práctica y discusión) y al tipo de agrupamientos. Sus resultados mostraron que ninguno de los tres enfoques de enseñanza analizados era asumido en su totalidad ni en exclusividad por ningún docente. Los enfoques más comunes fueron el enfoque de procesos, centrado en la enseñanza de la generación y organización de contenidos, así como de la revisión del texto, específicamente mediante la enseñanza de la generación de contenido del texto (90\% del profesorado), y el enfoque comunicativo, referido a la escritura de textos con un propósito real dirigidos a una audiencia concreta y su puesta en común para recibir feedback del lector, específicamente en relación a compartir el texto final con los compañeros (90\% del profesorado). Por su parte, el enfoque de instrucción estratégica, referido al uso de estrategias para enseñar el proceso de escritura ( $36 \%$ del profesorado) y al modelado de las mismas (40\% del profesorado), fue el menos implementado. En cuanto a las actividades de aula y los agrupamientos, la 
mayoría del tiempo se dedicó a la enseñanza directa por parte del profesorado a todo el grupo-aula y a la práctica del alumnado de forma individual.

Dichos estudios, pese a su naturaleza observacional, no ofrecen datos que vayan más allá del tipo de enfoque más comúnmente adoptado por el profesorado para la enseñanza de la escritura o del tiempo dedicado a diferentes contenidos, actividades, procedimientos instruccionales, agrupamientos y materiales. Por ello, las conclusiones ofrecidas no permiten alcanzar una comprensión profunda sobre cómo enseña el profesorado ciertas habilidades o procesos de escritura implicados en la composición textual, el tipo de conocimiento que se le proporciona al alumnado o la secuencia instruccional utilizada para ello.

En este sentido, se plantea el presente estudio de carácter exploratorio, que aborda, mediante la observación, el análisis en profundidad del proceso de enseñanza que sigue el profesorado en el grupo clase para que su alumnado adquiera el dominio de una habilidad compleja de la escritura como es la composición textual. Dicho estudio permitirá, en primer lugar, analizar qué procesos cognitivos implicados en el proceso de composición textual se enseñan, diferenciando entre aquellos de bajo nivel cognitivo o mecánicos, relacionados con la ortografía, la caligrafía y la gramática, - de alto nivel cognitivo o sustantivos, relacionados con la autorregulación de la planificación y revisión textual (Berninger y Winn, 2006). Asimismo, permitirá analizar qué tipo de conocimientos se trabajan dentro de la composición escrita, y principalmente su naturaleza declarativa, ligada al análisis de la tipología textual y sus características, o su naturaleza procedimental, ligada al proceso de escritura y su dominio estratégico y autorregulado. Al mismo tiempo, hará posible el análisis de qué procesos instruccionales utiliza el profesorado en su enseñanza de la escritura, así como la secuencia utilizada en la implementación de los mismos. En definitiva, todo ello permitirá evaluar el ajuste de la práctica del profesorado a las evidencias empíricas en relación a la enseñanza de la composición textual y en especial, al enfoque de instrucción estratégica y autorregulada, al ser ésta la práctica instruccional más efectiva para la mejora de la competencia escrita del alumnado (ver meta-análisis de Graham y Harris, 2018). Adicionalmente, se explora si la práctica implementada para la enseñanza de la composición textual difiere entre el profesorado atendiendo a ciertas variables moduladores del docente como su nivel de preparación, su eficacia personal y su actitud en relación a la enseñanza de la escritura; variables todas ellas que parecen influir en la práctica instruccional implementada por el profesorado para la enseñanza de la escritura (Rietdijk et al., 2018; Sánchez-Rivero et al., 2021).

\section{MÉTODO}

\section{PARTICIPANTES}

Participaron en el estudio siete docentes de dos centros educativos de la Provincia de León (España). Todos ellos tenían la titulación de Diplomado en Magisterio e impartían la asignatura de Lengua Castellana y Literatura en $4^{\circ}$ de Educación Primaria. Del total de participantes, 4 eran mujeres $(57,1 \%)$ y 3 hombres (42,9\%). La media de años de experiencia docente de los participantes en el estudio era de 19,71 (DT $=11,16)$, dedicando a su vez, como media, unos 82,86 minutos semanales a la enseñanza de la escritura (DT $=81,34)$.

\section{INSTRUMENTOS DE MEDIDA}

\section{INFORMACIÓN SOCIODEMOGRÁFICA Y NIVEL DE PREPARACIÓN PARA LA ENSEÑANZA DE LA ESCRITURA}

Se diseñó un cuestionario para recoger los datos descriptivos de los participantes relativos al género, los años de experiencia docente, el tiempo que dedicaban semanalmente a la enseñanza de la escritura y el nivel de preparación para la enseñanza de la escritura. Este último se evaluó a partir de 
dos ítems acerca del nivel de preparación que el profesorado consideraba haber obtenido para la enseñanza de la escritura durante su formación universitaria (ítem 1) y con posterioridad mediante cursos de especialización (ítem 2), con una escala de respuesta tipo Likert de cuatro alternativas: ninguna (1), mínima (2), adecuada (3) y alta (4). La puntuación total obtenida consiste en el promedio de las puntuaciones otorgadas a ambos ítems, pudiéndose obtener, por lo tanto, puntuaciones globales que oscilaban entre 2 y 8.

\section{CUESTIONARIO DE EFICACIA DOCENTE PERSONAL PARA LA ESCRITURA}

Se utilizó una traducción al castellano del cuestionario Teacher Efficacy Scale for Writing (Graham et al., 2001). El cuestionario consta de 10 ítems (ej. "Si el alumnado mejora su escritura, generalmente es porque he encontrado mejores formas de adaptar el método de enseñanza a cada alumno/a") centrados en evaluar las creencias del docente acerca de su capacidad para enseñar a escribir e influir positivamente en el rendimiento escritor del alumnado. Para su cumplimentación se utiliza una escala de respuesta tipo Likert que incluye las siguientes categorías: muy en desacuerdo (1), moderadamente en desacuerdo (2), ligeramente en desacuerdo (3), ligeramente de acuerdo (4), moderadamente de acuerdo (5) o muy de acuerdo (6). La puntuación total obtenida en esta dimensión consiste en el promedio de las puntuaciones otorgadas a los ítems que la constituyen de tal forma que, a mayor puntuación, mayor es el nivel de eficacia personal del profesorado, con un rango de puntuación total que varía desde 10 hasta 60.

CUESTIONARIO SOBRE LA ACTITUD DEL PROFESORADO HACIA LA ESCRITURA Y SU ENSEÑANZA

Se utilizó una traducción al castellano del cuestionario de Brindle et al. (2016), centrado en evaluar el grado de importancia que otorga el docente a la enseñanza de la escritura y a la habilidad de escribir. Consta de 7 ítems (ej. "Me gusta enseñar a escribir") con una escala de respuesta Likert con las siguientes categorías: muy en desacuerdo (1), moderadamente en desacuerdo (2), ligeramente en desacuerdo (3), ligeramente de acuerdo (4), moderadamente de acuerdo (5) o muy de acuerdo (6). La puntuación total obtenida en esta dimensión consiste en el promedio de las puntuaciones otorgadas a los ítems que la constituyen, con un rango de puntuación global que varía desde 7 hasta 42, reflejando una mayor puntuación, una actitud más positiva en el profesorado hacia la escritura y su enseñanza.

\section{SISTEMA DE CATEGORÍAS DE ANÁLISIS DE LA ENSEÑANZA DE LA COMPOSICIÓN TEXTUAL}

Se diseñó un sistema de categorías ad-hoc basado en la Guía Práctica sobre la enseñanza de la escritura en la Educación Primaria que recoge una serie de pautas, en base a las evidencias científicas existentes en el ámbito instruccional de la escritura, relacionadas no sólo con qué aspectos implicados en la composición textual se deben enseñar, sino cómo deben ser enseñados, elaborada por un equipo internacional de expertos (Graham et al., 2012). Así, en base a dicha guía se diseñó un sistema de categorías que consta de dos dimensiones de análisis.

La primera dimensión de análisis hace referencia a qué procesos y conocimientos implicados en la composición textual se enseñan, incluyendo las categorías y subcategorías de análisis que se recogen en la Tabla 1.

La segunda dimensión de análisis hace referencia a cómo se enseñan los diferentes procesos y conocimientos implicados en la composición textual, e incluye tres categorías de análisis, referidas a los procedimientos instruccionales empleados por el docente; los agrupamientos del alumnado; y los materiales. En la Tabla 2 se recogen y describen cada una de ellas, así como las subcategorías de análisis incluidas en su caso. 
Tabla 1

Categorías y subcategorías para el análisis de qué procesos y conocimientos de la composición textual se enseñan

\begin{tabular}{|c|c|}
\hline $\begin{array}{l}\text { Categoría/ } \\
\text { Subcategoría }\end{array}$ & Definición \\
\hline Planificación & $\begin{array}{c}\begin{array}{c}\text { Enseñar a establecer metas de escritura o a generar ideas y organizarlas en función del propósito } \\
\text { del texto. }\end{array}\end{array}$ \\
\hline $\begin{array}{l}\text { Establecimiento de } \\
\text { objetivos }\end{array}$ & $\begin{array}{c}\text { Proporcionar pautas/reglas que ayuden a comprender las condiciones de la tarea de escritura o los } \\
\text { pasos a seguir para su realización. Se diferencia entre objetivos vinculados: } \\
\text { Al proceso de escritura: mediante la enseñanza de estrategias que guíen el proceso de } \\
\text { composición textual. } \\
\begin{array}{c}\text { Al producto textual: pautas en relación a las características específicas de la tipología textual como } \\
\text { el objetivo comunicativo, la audiencia o la estructura textual. }\end{array} \\
\begin{array}{c}\text { A otras condiciones de la tarea de escritura ligadas a aspectos mecánicos del texto en relación a } \\
\text { su extensión y formato y/o a la ortografía y caligrafía. }\end{array}\end{array}$ \\
\hline Generación de ideas & $\begin{array}{l}\text { Enseñar al alumnado a generar ideas, por ejemplo, a partir de la lectura de diferentes fuentes, la } \\
\text { lluvia de ideas, la discusión. }\end{array}$ \\
\hline Organización de ideas & $\begin{array}{c}\text { Enseñar al alumnado a organizar el contenido del texto, por ejemplo, mediante la ordenación/ } \\
\text { esquematización de las ideas identificando ideas principales y secundarias. }\end{array}$ \\
\hline Redacción & $\begin{array}{c}\text { Enseñar a realizar una versión preliminar del texto en el que se traduzcan las ideas en lenguaje } \\
\text { escrito atendiendo a las características específicas de la tipología textual. Implica tanto la } \\
\text { enseñanza de las habilidades básicas o procesos de bajo nivel cognitivo de la escritura como la } \\
\text { enseñanza de las características específicas de la tipología textual. }\end{array}$ \\
\hline
\end{tabular}

Habilidades básicas o procesos de bajo nivel de la escritura

\begin{tabular}{|c|c|}
\hline Caligrafía & $\begin{array}{c}\text { Enseñar la caligrafía, por ejemplo, mediante la enseñanza del agarre del lápiz o el trazado de las } \\
\text { letras. }\end{array}$ \\
\hline Ortografía & $\begin{array}{l}\text { Enseñar la ortografía, por ejemplo, a partir de la enseñanza de las reglas ortográficas, el análisis } \\
\text { ortográfico de palabras derivadas o la búsqueda de palabras en el diccionario. }\end{array}$ \\
\hline Gramática & $\begin{array}{c}\text { Enseñar la gramática, por ejemplo, enseñando a construir oraciones atendiendo a la selección de } \\
\text { palabras, la sintaxis y la puntuación. }\end{array}$ \\
\hline \multicolumn{2}{|c|}{ Características de la tipología textual } \\
\hline $\begin{array}{l}\text { Enseñanza de } \\
\text { estrategias }\end{array}$ & $\begin{array}{l}\text { Enseñar estrategias que guíen el proceso de redacción considerando las características del texto } \\
\text { como, por ejemplo, el acrónimo IDC (Introducción, Desarrollo, Conclusión) + las vocales (Objetivo, } \\
\text { Audiencia, Ideas, Unir, Estructura). }\end{array}$ \\
\hline Objetivo comunicativo & $\begin{array}{l}\text { Enseñar el objetivo comunicativo de la tipología textual, por ejemplo, enfatizando el propósito del } \\
\text { texto o asociando el mismo a situaciones reales comunicativas. }\end{array}$ \\
\hline Audiencia & $\begin{array}{l}\text { Desarrollar/incrementar el concepto de audiencia, por ejemplo, escribiendo sobre el mismo tema } \\
\text { para diferentes audiencias o generando un listado de posibles audiencias y seleccionando la que } \\
\text { más se ajuste al tema. }\end{array}$ \\
\hline Estructura textual & $\begin{array}{l}\text { Enseñar la estructura o partes de la tipología textual y su elaboración en párrafos, por ejemplo, } \\
\text { mediante la observación y emulación de la estructura de modelos textuales ejemplares. }\end{array}$ \\
\hline
\end{tabular}


Tabla 1 (Continuación)

Categorías y subcategorías para el análisis de qué procesos y conocimientos de la composición textual se enseñan

\begin{tabular}{|c|c|}
\hline $\begin{array}{l}\text { Categoría/ } \\
\text { Subcategoría }\end{array}$ & Definición \\
\hline $\begin{array}{l}\text { Características } \\
\text { lingüísticas }\end{array}$ & $\begin{array}{l}\text { Enseñar las características lingüísticas específicas del texto, como por ejemplo, el tipo de } \\
\text { expresiones utilizadas, el uso de enlaces, la longitud y complejidad de las oraciones. }\end{array}$ \\
\hline Evaluación & $\begin{array}{c}\text { Enseñar a comprobar si el texto cumple los objetivos de escritura previamente establecidos } \\
\text { (evaluación criterial), por ejemplo, mediante el uso de listas de verificación o rúbricas para realizar } \\
\text { una autoevaluación o coevaluación del texto. }\end{array}$ \\
\hline Revisión & $\begin{array}{l}\text { Enseñar a hacer cambios de contenido en función de la evaluación previa como, por ejemplo, } \\
\text { mediante la adición, eliminación, sustitución u organización del contenido del texto. }\end{array}$ \\
\hline Edición & $\begin{array}{l}\text { Enseñar a revisar los aspectos ortográficos, gramaticales y de formato del texto, por ejemplo, } \\
\text { mediante su recuerdo o incluyendo estos aspectos en la evaluación criterial. }\end{array}$ \\
\hline Compartir el texto & $\begin{array}{c}\text { Supone la puesta en común del texto con los compañeros o el profesor, mediante su lectura y/o } \\
\text { escucha, para recibir feedback positivo o sugerencias de mejora. A diferencia del proceso de } \\
\text { Evaluación, no consiste en realizar una evaluación criterial sino en intercambiar opiniones acerca } \\
\text { de lo que les ha gustado o no del texto. }\end{array}$ \\
\hline
\end{tabular}

Tabla 2

Categorías y subcategorías para el análisis de cómo se enseña la composición textual

\begin{tabular}{|c|c|}
\hline $\begin{array}{l}\text { Categoría/ } \\
\text { Subcategoría }\end{array}$ & Definición \\
\hline $\begin{array}{l}\text { Procedimientos } \\
\text { instruccionales }\end{array}$ & $\begin{array}{c}\text { Hace referencia a los diferentes métodos que puede utilizar el profesorado para enseñar los } \\
\text { diferentes contenidos y/o estrategias de escritura. }\end{array}$ \\
\hline $\begin{array}{l}\text { Activación } \\
\text { conocimiento previo }\end{array}$ & Activar el conocimiento previo del alumnado acerca del contenido/estrategia a enseñar. \\
\hline $\begin{array}{l}\text { Motivación hacia la } \\
\text { tarea }\end{array}$ & $\begin{array}{c}\text { Proporcionar conocimiento acerca de la importancia del contenido/estrategia a enseñar y sus } \\
\text { beneficios. }\end{array}$ \\
\hline Enseñanza directa & $\begin{array}{c}\text { Explicación por parte del docente del contenido/estrategia. La enseñanza directa puede realizarse } \\
\text { mediante: } \\
\text { Presentación: mera exposición de la información sin ningún tipo de interacción con el alumnado. } \\
\text { Preguntas-Respuestas: explicación interactuando con el alumnado a través de la realización de } \\
\text { preguntas-respuestas. } \\
\text { Ejemplificación: apoyar la explicación con uno o varios ejemplos. }\end{array}$ \\
\hline Modelado & $\begin{array}{c}\text { Demostración por parte del docente de cómo realizar el proceso/estrategia, explicando/ } \\
\text { verbalizando en voz alta los pasos que está siguiendo. }\end{array}$ \\
\hline Práctica colaborativa & $\begin{array}{c}\text { El alumnado practica el contenido/estrategia enseñada en parejas o pequeño grupo y el profesor } \\
\text { proporciona ayudas según las necesidades detectadas. }\end{array}$ \\
\hline $\begin{array}{l}\text { Práctica individual } \\
\text { guiada }\end{array}$ & $\begin{array}{c}\text { El alumnado practica el contenido/estrategia de forma individual y el profesor proporciona ayudas/ } \\
\text { apoyos en caso necesario. }\end{array}$ \\
\hline
\end{tabular}


Tabla 2 (Continuación)

Categorías y subcategorías para el análisis de cómo se enseña la composición textual

\begin{tabular}{|c|c|}
\hline $\begin{array}{l}\text { Categoría/ } \\
\text { Subcategoría }\end{array}$ & Definición \\
\hline $\begin{array}{l}\text { Práctica } \\
\text { independiente }\end{array}$ & $\begin{array}{l}\text { El alumnado practica el contenido/estrategia sin ningún tipo de ayuda. El docente puede recordar } \\
\text { porqué y cuándo utilizarlo, pero no proporciona ayudas de cómo implementarla. }\end{array}$ \\
\hline $\begin{array}{l}\text { Transferencia del } \\
\text { aprendizaje }\end{array}$ & $\begin{array}{l}\text { El profesorado favorece la reflexión acerca de otras tareas o contextos en los que puedan aplicar } \\
\text { los contenidos/estrategias aprendidas. }\end{array}$ \\
\hline Otros & Otros procedimientos instruccionales no recogidos previamente. \\
\hline Agrupamientos & Hace referencia a las formas de agrupamiento del alumnado. \\
\hline Grupo-aula & Todo el alumnado. \\
\hline Gran grupo & Nueve o más alumnos. \\
\hline Pequeño grupo & Entre tres y ocho alumnos. \\
\hline \multicolumn{2}{|l|}{ Parejas } \\
\hline Individual & Puede ser con interacciones ocasionales o incidentales \\
\hline Materiales & $\begin{array}{c}\text { Consiste en una categoría de respuesta abierta para registrar todos aquellos materiales y recursos } \\
\text { que emplea el profesorado para la enseñanza de la composición textual. }\end{array}$ \\
\hline
\end{tabular}

\section{PROCEDIMIENTO}

En primer lugar, se contactó con el Equipo Directivo de los dos centros educativos para solicitar una reunión con su profesorado de Lengua Castellana y Literatura de $4^{\circ}$ de Educación Primaria con el fin de explicarles el propósito del estudio y solicitar su consentimiento de participación. Con el profesorado que aceptó formar parte del estudio, se realizó una reunión adicional individualizada para resolver todas las dudas y aclaraciones del estudio y su desarrollo, así como, para solicitar la cumplimentación de los cuestionarios descritos previamente. Asimismo, el Equipo Directivo de los centros educativos informaron sobre el desarrollo del estudio a las correspondientes familias del alumnado implicado en el mismo para su consentimiento.
A continuación, se realizó un registro audiovisual del número de sesiones que el profesorado consideró necesarias para enseñar a su grupo clase, tal como lo haría en su práctica habitual, la composición escrita de una tipología concreta de texto elegida libremente por ellos. De los 7 participantes en el estudio, cinco realizaron dos sesiones de enseñanza y dos realizaron una sesión de enseñanza por lo que, en total, se registraron 12 sesiones de grabación de una duración aproximada de 40 minutos cada una. Cabe señalar que uno de los profesores participantes (Profesor 3), si bien realizó dos sesiones de enseñanza, en cada una de ellas enseñó una tipología textual diferente, por lo que dichas sesiones de grabación se analizaron de forma independiente. En la Tabla 3 se presenta la tipología textual enseñada por cada profesor, así como el número de sesiones empleadas y su duración total. 
Tabla 3

Tipología textual enseñada, número de sesiones y duración total.

\begin{tabular}{|c|c|c|c|c|c|c|c|c|}
\hline & Profesor 1 & Profesor 2 & \multicolumn{2}{|c|}{ Profesor 3 } & Profesor 4 & Profesor 5 & Profesor 6 & Profesor 7 \\
\hline Tipología & Receta & Receta & Receta & E-mail & E-mail & Cuento & Cuento & Cuento \\
\hline No sesiones & 2 & 2 & 1 & 1 & 1 & 2 & 1 & 2 \\
\hline $\begin{array}{c}\text { Tiempo } \\
\text { total } \\
\text { (minutos) }\end{array}$ & 84 & 72 & 44 & 45 & 44 & 96 & 40 & 61 \\
\hline
\end{tabular}

Las tipologías textuales elegidas por el profesorado respondieron a diferentes funciones comunicativas (ver Tabla 3). Así, en tres casos se enseñó un tipo de texto narrativo como el cuento cuya función consiste en informar sobre acciones o sucesos, y en otras tres ocasiones se trabajó un tipo de texto directivo o instructivo como la receta con una función de dirigir, ordenar o aconsejar (Loureda, 2009). En dos casos, se enseñó un tipo de texto conversacional como el e-mail que responde a nuevas formas de comunicación a través de medios electrónicos (Alexopoulou, 2010) y que puede cumplir diversas funciones comunicativas con un marcado carácter interactivo (Loureda, 2009).

Las sesiones de grabación se establecieron a conveniencia del profesorado, dentro del horario escolar y tuvieron lugar en el segundo semestre del curso, aproximadamente, al mismo tiempo para toda la muestra. Durante los registros audiovisuales se evitó el registro de imágenes reconocibles del alumnado, tomándose siempre la imagen desde el fondo de la clase y dirigida al registro de la pizarra. Asimismo, se aseguró el anonimato del profesorado participante y se cumplió con los acuerdos de confidencialidad y ajuste al código deontológico que debe regir toda investigación.

\section{ANÁLISIS DE DATOS}

Para analizar la información obtenida a partir de las observaciones, se siguió una metodología cualitativa mediante un análisis de contenido a través del software MAXQDA
Analytics Pro 2020 (VERBI Software, 2019). Para ello, se procedió a realizar las trascripciones en Word de cada uno de los registros audiovisuales realizados y se importaron al software MAXQDA para proceder a la lectura, análisis y codificación de las mismas. Respecto a las variables moduladoras del docente relativas a su preparación, su eficacia personal y su actitud en relación a la enseñanza de la escritura, se calcularon estadísticos descriptivos como la media y la desviación típica con el fin de analizar si las puntuaciones obtenidas en estas variables eran similares entre el profesorado, o si, por el contrario, profesores que puntuaran diferente en las mismas, implementaban una práctica de enseñanza diferenciada a la del resto de profesores.

\section{RESULTADOS}

En primer lugar, se presentan los resultados relativos al análisis de la enseñanza de la composición textual. Cabe señalar que, en todos los casos analizados, se identificó una estructura común de la/s sesión/es de enseñanza en dos partes claramente diferenciadas. Una primera parte dedicada a la enseñanza propiamente dicha de los diferentes procesos y conocimientos de la escritura y una segunda parte dedicada a la práctica de la escritura del texto por parte del alumnado y a la evaluación del mismo. En base a ello, en este primer apartado, se presentan en primer lugar, los resultados del análisis de la enseñanza de la composición textual en 
relación a qué procesos se enseñan y cómo se enseñan, y posteriormente, se presentan los resultados relativos al tipo de práctica que realiza el alumnado (colaborativa, guiada o independiente), y en su caso, el tipo de andamiaje proporcionado por el profesorado, así como el tipo de evaluación del texto realizada y si esta se centra en aspectos sustantivos o superficiales del texto. Finalmente, se presenta un segundo apartado en el que se muestran los resultados del análisis de las variables moduladoras del docente y su posible relación con la práctica de enseñanza implementada.

\section{RESULTADOS DEL ANÁLISIS DE QUÉ PROCESOS Y CONOCIMIENTOS SOBRE LA COMPOSICIÓN TEXTUAL SE ENSEÑAN Y CÓMO SE ENSEÑAN}

Como se muestra en la Tabla 4, en la enseñanza de la composición escrita, de los siete profesores analizados, cuatro trabajaron la planificación textual a partir del establecimiento de objetivos ligados exclusivamente al conocimiento del producto textual, bien a nivel sustantivo (propósito del texto, audiencia, estructura y su elaboración en párrafos y elementos a incluir) o bien a nivel mecánico (presentación, extensión y formato del texto). En ningún caso se observó el establecimiento de objetivos vinculados al proceso de escritura, ni ningún tipo de instrucción estratégica en torno a cómo planificar. Sin embargo, no todos los profesores que utilizaron el establecimiento de objetivos vinculados al producto textual enseñaron específicamente los mismos contenidos. Así, el Profesor 5 se focalizó exclusivamente en los elementos a incluir en el cuento y en aspectos de extensión y presentación del texto. Por su parte el Profesor 3 (en el caso de la receta) estableció objetivos relacionados sólo con la estructura y la audiencia del texto, mientras que los Profesores 6 y 7 , además de incluir objetivos relacionados con los aspectos mecánicos del texto, su estructura y los elementos a incluir en el cuento, también incluyeron el propósito $u$ objetivo comunicativo del mismo. A su vez, sólo el Profesor 7 trabajó la generación de ideas dentro de la planificación textual, mientras que la organización de las ideas del texto en su planificación no fue enseñada por ninguno de los profesores analizados.

En cuanto a cómo se enseñaron estos contenidos de planificación textual, se observaron dos procedimientos instruccionales diferenciados, la activación de conocimientos previos y la enseñanza directa, esta última, bien a través de una mera exposición sin ningún tipo de interacción con el alumnado (presentación), bien apoyada en la ejemplificación o bien a través de preguntas-respuestas (ver Tabla 4). Así, el Profesor 5 realizó una simple activación de conocimientos previos, como paso previo y único a la escritura del texto por parte del alumnado, a partir de una lluvia de ideas con el alumnado en la que se establecieron los objetivos de escritura en relación a los diferentes elementos a incluir en el cuento y a los aspectos mecánicos del texto (extensión y presentación). Por su parte, los profesores 6 y 7 utilizaron la enseñanza directa para establecer los objetivos de escritura vinculados al producto textual, si bien el Profesor 6 realizó una mera exposición de la información con una escasa interacción con el alumnado (presentación), mientras que el Profesor 7 apoyó la enseñanza directa con la ejemplificación, tanto para ejemplificar, en primer lugar, los objetivos establecidos a partir de cuentos conocidos como el de "Caperucita Roja" como para enseñar, tras la presentación de los objetivos de escritura, la generación de ideas mediante la proyección en la pizarra de unas viñetas a partir de las cuales el alumnado debía escribir el cuento. Finalmente, el Profesor 3 combinó ambos procedimientos instruccionales, realizando en primer lugar, una activación de conocimientos previos acerca de las fases de la escritura (planificación, redacción, evaluación) y a continuación, una enseñanza directa, mediante la realización de preguntas-respuestas, focalizada en el tipo de texto, su estructura y la audiencia.

Cabe señalar que, el resto de profesores, si bien no trabajaron de forma explícita la planificación textual, estos aludieron de forma puntual, antes de iniciarse la práctica de escritura del texto por parte del alumnado, a la necesidad de dedicar un tiempo a pensar y organizar las ideas. 
Tabla 4

Resultados del análisis de la enseñanza del proceso de planificación

\begin{tabular}{|r|c|c|c|c|c|c|c|c|c|}
\hline Profesor & 1 & 2 & $\begin{array}{c}3 \\
\text { (receta) }\end{array}$ & $\begin{array}{c}3 \\
\text { (e-mail) }\end{array}$ & 4 & 5 & 6 & 7 & $\begin{array}{c}\text { Número } \\
\text { Total }\end{array}$ \\
\hline
\end{tabular}

Planificación

\begin{tabular}{|l|c|c|c|c|c|c|c|c|c|}
\hline $\begin{array}{l}\text { Establecimiento } \\
\text { objetivos }\end{array}$ & - & - & 1 & - & - & 1 & 1 & 1 & 4 \\
\hline Generación de ideas & - & - & - & - & - & - & - & 1 & 1 \\
\hline $\begin{array}{l}\text { Organización de } \\
\text { ideas }\end{array}$ & - & - & - & - & - & - & - & - & 0 \\
\hline
\end{tabular}

Cómo se enseña (Procedimientos instruccionales)

\begin{tabular}{|l|c|c|c|c|c|c|c|c|c|}
\hline $\begin{array}{l}\text { Activación } \\
\text { conocimiento previo }\end{array}$ & - & - & 1 & - & - & 1 & - & - & 2 \\
\hline $\begin{array}{l}\text { Motivación hacia } \\
\text { tarea }\end{array}$ & - & - & - & - & - & - & - & - & 0 \\
\hline
\end{tabular}

Enseñanza directa:

\begin{tabular}{|l|c|c|c|c|c|c|c|c|c|}
\hline Presentación & - & - & - & - & - & - & 1 & - & 1 \\
\hline Ejemplificación & - & - & - & - & - & - & - & 1 & 1 \\
\hline Pregunta-respuesta & - & - & 1 & - & - & - & - & - & 1 \\
\hline Modelado & - & - & - & - & - & - & - & - & 0 \\
\hline Práctica colaborativa & - & - & - & - & - & - & - & - & 0 \\
\hline $\begin{array}{l}\text { Práctica individual } \\
\text { guiada }\end{array}$ & - & - & - & - & - & - & - & - & 0 \\
\hline $\begin{array}{l}\text { Práctica } \\
\text { independiente }\end{array}$ & - & - & - & - & - & - & - & - & 0 \\
\hline $\begin{array}{l}\text { Transferencia del } \\
\text { aprendizaje }\end{array}$ \\
$\begin{array}{l}\text { Nota. - no lo enseñaron/emplearon; } 1=\text { sí lo enseñaron/emplearon; número total = número total de profesores que lo } \\
\text { enseñaron/emplearon. }\end{array}$ \\
\hline
\end{tabular}

En relación al proceso de redacción, como se puede observar en la Tabla 5, en ningún caso se observó la enseñanza específica de los procesos de bajo nivel cognitivo de la escritura, como la caligrafía, la ortografía y la gramática. Tampoco se observó en ningún caso analizado la enseñanza de estrategias vinculadas al proceso de redacción. El objetivo comunicativo del texto, sólo fue enseñado por dos profesores, enfatizando el propósito del mismo y presentando como tarea a realizar por el alumnado, una tarea de escritura con un propósito real dirigida a una audiencia concreta. Asimismo, sólo estos dos profesores enseñaron la audiencia del texto mediante la adecuación del saludo y la despedida del e-mail al lector. Finalmente, la estructura del texto y su elaboración, así como 
las características lingüísticas del mismo (tipo de expresiones, tipo de enlaces utilizados, longitud de las oraciones), fueron enseñadas por cuatro profesores.

Respecto a los procedimientos instruccionales empleados para la enseñanza de los contenidos trabajados en la redacción textual, se observó una mayor variabilidad en relación a los utilizados para enseñar la planificación textual. Así, si bien la enseñanza directa, en este caso apoyada en la ejemplificación, fue, de nuevo, el procedimiento instruccional más utilizado para enseñar tanto el propósito del texto y su audiencia, como la estructura textual y las características lingüísticas del texto, también se emplearon otros procedimientos instruccionales, no usados hasta ahora, y que resultan más eficaces en base a estudios empíricos, como el análisis de modelos textuales y el modelado (ver Tabla 5). Estos procedimientos instruccionales además favorecen en mayor medida el proporcionar un conocimiento procedimental del proceso de redacción. Ambos procedimientos instruccionales fueron utilizados exclusivamente para enseñar la estructura textual y su elaboración, así como las características lingüísticas del texto, si bien el análisis de modelos textuales fue utilizado por tres profesores, mientras que el modelado sólo fue utilizado por un profesor (ver Tabla 5).

A su vez, se observó cierta variabilidad entre el profesorado en relación a la secuencia instruccional empleada para enseñar los contenidos trabajados en la redacción textual, aunque en todos los casos se comenzó proporcionando una enseñanza directa, bien apoyada en el análisis de modelos textuales (Profesores 1, 2 y 3) o bien apoyada en la ejemplificación (Profesores 3 y 4). Asimismo, en relación a los tres profesores que proporcionaron una enseñanza directa apoyada en el análisis de modelos textuales, uno de ellos (Profesor 1) sólo utilizó este procedimiento instruccional, tanto para enseñar, a lo largo de la primera sesión de enseñanza, la estructura del texto y las características lingüísticas del mismo, como para recordar y recapitular los aspectos enseñados al inicio de la segunda sesión y antes de iniciar la práctica de escritura por parte del alumnado. Los otros dos profesores combinaron este procedimiento instruccional con la enseñanza directa apoyada en la ejemplificación (Profesor 3 en el caso de la receta) o con el modelado (Profesor 2). Así, el Profesor 3, primero proporcionó una enseñanza directa apoyada en el análisis de modelos textuales para enseñar exclusivamente la estructura del texto, y, a continuación, proporcionó una enseñanza directa apoyada en ejemplos para enseñar la elaboración del texto en párrafos y las características lingüísticas del mismo. Por su parte, el Profesor 2, proporcionó una enseñanza directa apoyada en el análisis de modelos textuales para enseñar, a lo largo de la primera sesión de enseñanza, la estructura del texto y su elaboración. Posteriormente, en la segunda sesión de enseñanza, volvió a utilizar este procedimiento instruccional para enseñar en este caso, las características lingüísticas del texto, y finalmente, y antes de iniciar la práctica de escritura por parte del alumnado, realizó un modelado de la escritura de una receta, verbalizando en voz alta los pasos que iba siguiendo e interactuando con el alumnado. Respecto a los Profesores 3 (en el caso del e-mail) y 4, si bien ambos comenzaron proporcionando una enseñanza directa apoyada en la ejemplificación para enseñar tanto el objetivo comunicativo y la audiencia del texto como la estructura del texto y las características lingüísticas del mismo, el Profesor 3, tras dicha enseñanza realizó una recapitulación de los aspectos enseñados a partir de nuevos ejemplos y antes de iniciar la práctica de escritura por parte del alumnado, mientras que el Profesor 4, introdujo una práctica individual guiada tras cada módulo de enseñanza directa.

En cuanto al proceso de evaluación del texto, sólo uno de los siete profesores (ver Tabla 6) enseñó al alumnado a realizar una evaluación criterial del texto escrito a partir de una rúbrica de evaluación centrada en la comprobación de la estructura textual, del sentido y orden del contenido y de aspectos ortográficos. Para ello, como se muestra en la 


\section{Tabla 5}

Resultados del análisis de la enseñanza del proceso de redacción

\begin{tabular}{|c|c|c|c|c|c|c|c|c|c|}
\hline Qué se enseña & 1 & 2 & $\begin{array}{c}3 \\
\text { (receta) }\end{array}$ & $\begin{array}{c}3 \\
\text { (e-mail) }\end{array}$ & 4 & 5 & 6 & 7 & $\begin{array}{l}\text { Número } \\
\text { Total }\end{array}$ \\
\hline \multicolumn{10}{|l|}{ Redacción } \\
\hline Caligrafía & - & - & - & - & - & - & - & - & 0 \\
\hline Ortografía & - & - & - & - & - & - & - & - & 0 \\
\hline Gramática & - & - & - & - & - & - & - & - & 0 \\
\hline Estrategias & - & - & - & - & - & - & - & - & 0 \\
\hline $\begin{array}{l}\text { Objetivo } \\
\text { comunicativo }\end{array}$ & - & - & 1 & 1 & 1 & - & - & - & 2 \\
\hline Audiencia & - & - & - & 1 & 1 & - & - & - & 2 \\
\hline Estructura textual & 1 & 1 & 1 & 1 & 1 & - & - & - & 4 \\
\hline $\begin{array}{l}\text { Características } \\
\text { lingüísticas }\end{array}$ & 1 & 1 & 1 & 1 & 1 & - & - & - & 4 \\
\hline
\end{tabular}

Cómo se enseña (Procedimientos instruccionales)

\begin{tabular}{|l|c|c|c|c|c|c|c|c|c|}
\hline $\begin{array}{l}\text { Activación } \\
\text { conocimiento previo }\end{array}$ & - & - & - & - & - & - & - & - & 0 \\
\hline $\begin{array}{l}\text { Motivación hacia } \\
\text { tarea }\end{array}$ & - & - & - & - & - & - & - & - & 0 \\
\hline
\end{tabular}

Enseñanza directa:

\begin{tabular}{|c|c|c|c|c|c|c|c|c|c|}
\hline Modelos textuales & 1 & 1 & 1 & - & - & - & - & - & 3 \\
\hline Presentación & - & - & - & - & - & - & - & - & 0 \\
\hline Ejemplificación & - & - & 1 & 1 & 1 & - & - & - & 2 \\
\hline Pregunta-respuesta & - & - & - & - & - & - & - & - & 0 \\
\hline Modelado & - & 1 & - & - & - & - & - & - & 1 \\
\hline Práctica colaborativa & - & - & - & - & - & - & - & - & 0 \\
\hline $\begin{array}{l}\text { Práctica individual } \\
\text { guiada }\end{array}$ & 1 & - & 1 & 1 & 1 & - & - & - & 3 \\
\hline $\begin{array}{l}\text { Práctica } \\
\text { independediente }\end{array}$ & - & 1 & - & - & - & 1 & 1 & 1 & 4 \\
\hline $\begin{array}{l}\text { Transferencia del } \\
\text { aprendizaje }\end{array}$ & - & & - & - & - & & - & & 0 \\
\hline
\end{tabular}

Nota. - = no lo enseñaron/emplearon; 1 = sí lo enseñaron/emplearon; número total = número total de profesores que lo enseñaron/emplearon. 
Tabla 6, proporcionó una enseñanza directa mediante la presentación, centrada tanto en el conocimiento declarativo (en qué consiste la rúbrica) como procedimental (cómo utilizarla para comprobar esos criterios en el propio texto y para puntuar los textos de los compañeros) del proceso de evaluación. Sin embargo, sorprendentemente, no se observó posteriormente la práctica por parte del alumnado de este proceso tras la realización del texto.

Respecto al proceso de revisión del texto, en ningún caso analizado se observó la enseñanza de estrategias ligadas al proceso de revisión ni de otro tipo de conocimientos relacionados con la revisión de los aspectos sustantivos o de contenido del texto, por ejemplo, a partir de técnicas específicas de adición, eliminación o sustitución de contenido. Sin embargo, tres profesores (Profesor 4, 5 y 7) aludieron, de forma puntual, a la necesidad de leer y revisar la estructura y la ortografía del texto una vez finalizada su escritura. En relación a la edición textual (aspectos superficiales de ortografía, presentación y formato) sólo fue enseñada por el Profesor 3 mediante la inclusión de este criterio en la rúbrica de evaluación del texto (ver Tabla 6).

Tabla 6

Resultados del análisis de la enseñanza de los procesos de evaluación, revisión y edición textual

\begin{tabular}{|l|c|c|c|c|c|c|c|c|c|}
\hline & Profesor & 1 & 2 & $\begin{array}{c}3 \\
\text { (receta) }\end{array}$ & $\begin{array}{c}3 \\
\text { Qué se enseña }\end{array}$ & 4 & 5 & 6 & $\begin{array}{c}\text { Número } \\
\text { Total }\end{array}$ \\
\hline Evaluación & - & - & 1 & - & - & - & - & - & 1 \\
\hline Revisión & - & - & - & - & - & - & - & - & 0 \\
\hline Edición & - & - & 1 & - & - & - & - & - & 1 \\
\hline
\end{tabular}

Cómo se enseña (Procedimientos instruccionales)

\begin{tabular}{|l|c|c|c|c|c|c|c|c|c|}
\hline Activación conocimiento previo & - & - & - & - & - & - & - & - & 0 \\
\hline Motivación hacia tarea & - & - & - & - & - & - & - & - & 0 \\
\hline
\end{tabular}

Enseñanza directa:

\begin{tabular}{|l|c|c|c|c|c|c|c|c|c|}
\hline Presentación & - & - & 1 & - & - & - & - & - & 1 \\
\hline Ejemplificación & - & - & - & - & - & - & - & - & 0 \\
\hline Pregunta-respuesta & - & - & - & - & - & - & - & - & 0 \\
\hline Modelado & - & - & - & - & - & - & - & - & 1 \\
\hline Práctica colaborativa & - & - & - & - & - & - & - & - & 0 \\
\hline Práctica individual guiada & - & - & - & - & - & - & - & - & 0 \\
\hline Práctica independediente & - & - & - & - & - & - & - & - & 0 \\
\hline Transferencia del aprendizaje & - & - & - & - & - & - & - & - & 0 \\
\hline Nota.
\end{tabular}

Nota. - = no lo enseñaron/emplearon; 1 = sí lo enseñaron/emplearon; número total = número total de profesores que lo enseñaron/emplearon. 
Por último, en relación a los agrupamientos y materiales empleados, en todos los casos analizados se enseñó a nivel de grupo-aula y únicamente se emplearon el libro de texto, la pizarra y el proyector. En ningún caso se observó el uso de recursos tecnológicos, ni tan siquiera en los casos en los que la tipología textual enseñada fue el correo electrónico.

Una vez finalizada la parte de enseñanza por parte del profesorado, en todos los casos, se continuó con una práctica individual del alumnado de la escritura del texto, bien de forma guiada o bien de forma independiente (ver Tabla 5). En los casos en los que se realizó una práctica guiada, los andamiajes proporcionados se centraron fundamentalmente en aspectos de estructura y contenido del texto, por ejemplo, proporcionándoles ideas a incluir en el texto, aunque también se proporcionaron ayudas enfocadas a la construcción de frases y a la selección de vocabulario.

Una vez finalizada la escritura del texto, tres profesores favorecieron el compartir el texto con los compañeros, bien mediante la lectura del propio texto (Profesor 5) o del de otro compañero (Profesores 6 y 7), sin realizar ningún tipo de evaluación criterial del mismo y limitando el feedback proporcionado a aspectos de fluidez lectora (ej. Tienes que leer más despacio) o de extensión del texto (ej. Este texto es muy cortito) o incluso sin ningún tipo de feedbak (ej. Bien, que lea el siguiente). Por su parte, sólo los Profesores 1 y 4 evaluaron los textos de su alumnado indicándoles, principalmente, los errores cometidos a nivel de ortografía y gramática, aunque también de estructura y sentido del texto.

Respecto a los materiales para la escritura del texto, se emplearon únicamente folios o el cuaderno del alumnado.

\section{RESULTADOS DEL ANÁLISIS DE LAS VARIABLES DEL DOCENTE MODULADORAS DE LA ENSEÑANZA DE LA ESCRITURA}

Como se puede observar en la Tabla 7, en general, el profesorado participante en este estudio muestra, en relación a la enseñanza de la escritura, una mínima preparación, una eficacia personal moderada y una actitud moderadamente positiva. Asimismo, a excepción del Profesor 1, se observan puntuaciones similares entre el profesorado en todas las variables consideradas, situándose éstas en torno a la media (ver Tabla 7). En el caso del Profesor 1 , si bien se observan puntuaciones inferiores, respecto al resto de profesores, en el nivel de eficacia personal y en la actitud en relación a la enseñanza de la escritura, no se observa un patrón diferenciado del resto de profesores en relación a la práctica de enseñanza de la composición textual que implementa.

\section{Tabla 7}

Descriptivos sobre la preparación, la eficacia personal y la actitud del profesorado en relación a la enseñanza de la escritura.

\begin{tabular}{|c|c|c|c|}
\hline & $\begin{array}{l}\text { Preparación } \\
\text { (Rango 1-4) }\end{array}$ & $\begin{array}{l}\text { Eficacia personal } \\
\text { (Rango 1-6) }\end{array}$ & $\begin{array}{c}\text { Actitud } \\
\text { (Rango 1-6) }\end{array}$ \\
\hline Profesor 1 & 2 & 3,70 & 3,57 \\
\hline Profesor 2 & 2 & 4,60 & 4,43 \\
\hline Profesor 3 & 2,5 & 4,90 & 4,14 \\
\hline Profesor 4 & 2,5 & 4,60 & 4,86 \\
\hline Profesor 5 & 2 & 4,80 & 5,14 \\
\hline Profesor 6 & 3 & 4,20 & 5,14 \\
\hline Profesor 7 & 2 & 4,10 & 5,29 \\
\hline Media (DT) & $2,29(0,39)$ & $4,41(0,43)$ & $4,65(0,63)$ \\
\hline
\end{tabular}




\section{DISCUSIÓN}

El presente estudio aborda, mediante la observación, el análisis de cómo el profesorado de $4^{\circ}$ de Educación Primaria enseña a su alumnado la composición de textos escritos, incluyendo el análisis de qué procesos y conocimientos se enseñan y cómo se enseñan. En base a los resultados obtenidos, es posible extraer las siguientes conclusiones.

En primer lugar, no se observó en el profesorado participante la enseñanza específica de los procesos de bajo nivel cognitivo de la escritura, como la caligrafía, la ortografía y la gramática. Esto parece confirmar que, la enseñanza de los procesos de bajo nivel cognitivo de la escritura como foco principal de instrucción, se da en los primeros cursos de Primaria, tal y como señalan las evidencias empíricas a nivel observacional sobre la enseñanza de la escritura en los primeros cursos de Educación Primaria (ver Coker et al., 2016). Sin embargo, en cursos superiores de Primaria, los procesos de bajo nivel cognitivo parecen dejar de ser el foco central de instrucción. No obstante, dicha conclusión podría verse condicionada por las propias características impuestas en el diseño del presente estudio, en el que se pedía al profesorado que enseñara la composición escrita de una tipología concreta de texto elegida por ellos. No en vano, estudios previos de encuesta realizados en España sobre la práctica de la enseñanza de la escritura (ver Sánchez-Rivero et al., 2021), han mostrado que el profesorado de los últimos cursos de Educación Primaria indica una mayor frecuencia de enseñanza de los procesos de bajo nivel cognitivo frente a los procesos de alto nivel cognitivo de la escritura. En línea con dicha conclusión, el profesorado del presente estudio, aunque no enseñó específicamente procesos de bajo nivel cognitivo, sí se consideraron, en la mayoría de los casos, aspectos relacionados con la ortografía y caligrafía del texto, bien como objetivos establecidos en la planificación textual o bien en la evaluación del texto por parte del docente; lo que indica que el profesorado sí otorgó importancia a estos aspectos en el producto textual final, aunque no los enseñara explícitamente, al menos en esta tarea de escritura.

En segundo lugar, en relación a los procesos de alto nivel cognitivo de la escritura, el profesorado analizado dio un mayor énfasis a la enseñanza del proceso de redacción ligado a las características específicas de la tipología textual, seguido del proceso de planificación, y una mínima o nula enseñanza de los procesos de evaluación y revisión textual; resultado coherente con lo obtenido por estudios previos a nivel internacional (Coker et al., 2016; Rietdijk et al., 2018). A su vez, ninguno de los procesos de alto nivel cognitivo de la escritura fue enseñado a través de una instrucción estratégica ligada a proporcionar un conocimiento procedimental y autorregulado del proceso de escritura, proporcionado, en la mayoría de los casos, un simple conocimiento declarativo ligado al producto textual. Este resultado si bien es coherente con los resultados obtenidos en el estudio de Rietdijk et al. (2018), contradice parcialmente los resultados obtenidos en el estudio de encuesta desarrollado en el contexto educativo español (Sánchez-Rivero et al., 2021), en el que el profesorado analizado indicó enseñar estrategias de planificación y de revisión textual con mayor frecuencia que la estructura de las diferentes tipologías textuales. Una posible explicación podría estar ligada a la conceptualización del profesorado sobre qué implica el proporcionar al alumnado una instrucción estratégica en escritura o lo que realmente supone el enseñar estrategias de planificación, redacción y revisión textual. En este sentido, si bien es cierto que el profesorado analizado enseñó aspectos claves del proceso de planificación textual, y en menor medida, del proceso de evaluación del texto, no lo hizo de una forma efectiva mediante la adopción de un enfoque estratégico, sino que sólo proporcionó una mera enseñanza directa; procedimiento instruccional que no resulta clave para la mejora del rendimiento escritor del alumnado (Fidalgo et al., 2011). Así, la nula o mínima enseñanza centrada en el dominio estratégico y autorregulado de los 
procesos de planificación y revisión textual podría explicar, en parte, el bajo rendimiento en composición escrita que muestra el alumnado de nuestro país (Ministerio de Educación, 2010) dado que ésta requiere no sólo el dominio del proceso de producción textual, sino, además, de la autorregulación de los procesos de planificación y revisión del texto por parte del alumnado (Berninger y Winn, 2006). Por tanto, se señala la necesidad de que el profesorado, además de enseñar habilidades de redacción textual, favorezca en mayor medida la autorregulación de los procesos de planificación y revisión, ya que estos procesos cognitivos no se desarrollan de forma espontánea (López et al., 2019).

En tercer lugar, en relación a los procedimientos instruccionales cabe extraer las siguientes conclusiones. El procedimiento instruccional más empleado por el profesorado analizado fue la enseñanza directa, bien apoyada en ejemplos o bien apoyada en el análisis de modelos textuales; resultados que van en línea con los obtenidos por estudios previos a nivel internacional (Coker et al., 2016; Rietdijk et al., 2018). El procedimiento de análisis de modelos textuales es una práctica instruccional que se ha corroborado efectiva para la mejora de la competencia escrita del alumnado (Graham y Harris, 2018). Sin embargo, en coherencia con los resultados obtenidos por el estudio de encuesta desarrollado en nuestro país (Sánchez-Rivero et al., 2021), el uso que el profesorado hace de este procedimiento instruccional no es muy frecuente. Por otra parte, un procedimiento instruccional altamente efectivo en la enseñanza de la escritura es el modelado. En este, el alumnado observa a un modelo realizando el proceso de escritura y explicando el proceso de pensamiento seguido en voz alta. El modelado cuenta con evidencias empíricas que apoyan su eficacia en el rendimiento escritor del alumnado, sobre todo cuando la observación va seguida de la reflexión por parte del alumnado de lo observado (Braaksma et al., 2002; Fidalgo et al., 2015). Sin embargo, es posible concluir que apenas se observó el uso del modelado por parte del profesorado analizado para la enseñanza de la composición textual.
De igual forma, tampoco se observó, en ningún caso analizado, el aprendizaje colaborativo en el proceso de escritura del texto ya que en todos los casos el alumnado realizó la tarea de composición textual de forma individual sin ningún tipo de interacción con sus compañeros. Este resultado está en línea con los estudios observacionales desarrollados a nivel internacional (Coker et al., 2016; Rietdijk et al., 2018), pero es contradictorio a los resultados obtenidos en el estudio de encuesta desarrollado en nuestro país (Sánchez-Rivero et al., 2021), que muestra que la escritura colaborativa es una práctica relativamente frecuente. En este sentido, el trabajo colaborativo en torno al proceso de escritura es una práctica que se ha corroborado efectiva para la mejora del rendimiento escritor del alumnado en los estudios de meta-análisis (Graham y Harris, 2018; Koster et al., 2015), favoreciendo la autorregulación del aprendizaje a través del aprendizaje vicario (Panadero y Alonso-Tapia, 2014) y la calidad de los textos producidos (De Smedt et al., 2020; Robledo et al., 2016).

Finalmente, respecto a los materiales utilizados, cabe señalar la ausencia de las tecnologías tanto para la enseñanza de la escritura como para la escritura del texto por parte del alumnado; resultado nuevamente acorde con los obtenidos por estudios previos tanto observacionales como de encuesta (Rietdiik et al., 2018; Sánchez-Rivero et al., 2021). Si bien diferentes estudios de metaanálisis (Graham y Harris, 2018) y de revisión (De Smedt y Van Keer, 2014) han mostrado los beneficios de introducir las nuevas tecnologías en la enseñanza de la escritura, su integración efectiva en el aula requeriría dotar al profesorado de una mejor infraestructura y de una mayor formación práctica en la creación de entornos virtuales ya que las principales dificultades que manifiestan en relación a esta cuestión son la falta de tiempo, recursos y formación (Sánchez y Galindo, 2018).

En definitiva, los resultados del presente estudio muestran que, al igual que sucede en la enseñanza de la lectura (Sánchez-Rivero y Fidalgo, 2020) el profesorado analizado, en general, no implementa, para la enseñanza de 
la escritura, una práctica instruccional basada en la evidencia empírica, con las consecuencias negativas que ello puede suponer en el rendimiento escritor del alumnado y en la detección de las dificultades en su aprendizaje en línea con el enfoque preventivo propuesto por el modelo de respuesta a la intervención (Arrimada et al., 2018; Ciullo et al., 2016; Crespo et al., 2013; Gottheil et al., 2019).

Por lo tanto, parece clave el proporcionar al profesorado programas de desarrollo profesional centrados en la enseñanza de la escritura, y en especial, en la implementación de una instrucción eficaz basada en evidencias empíricas. De entre ellas, cabe hacer una mención especial a la necesidad de favorecer un dominio del profesorado de la instrucción estratégica y autorregulada, totalmente ausente en la enseñanza del profesorado, pese a ser el enfoque instruccional más eficaz para la mejora de la competencia escrita del alumnado (ver Graham y Harris, 2018; Koster et al., 2015). Además, se cuenta con diversos estudios que han mostrado su efectividad en la mejora de la competencia escrita con alumnado de la Educación Primaria de cursos iniciales (Arrimada et al., 2019) o superiores (Fidalgo et al., 2015; Fidalgo et al., 2011; Torrance et al., 2007), de la Educación Secundaria Obligatoria (De la Paz y Graham, 2002), incluso con alumnado universitario (Robledo et al., 2018). Este enfoque instruccional combina el uso de diferentes estrategias y procedimientos instruccionales que favorecen la autorregulación del proceso escritor incluyendo el uso de reglas nemotécnicas, el modelado con pensamiento en voz alta y la práctica colaborativa e individual (Torrance et al., 2007).

Antes de finalizar, cabe reconocer que las conclusiones ofrecidas en el presente estudio deben considerarse dentro de los límites impuestos por las características de la muestra observada en relación a la generalización de los resultados obtenidos. Así, si bien los resultados se ajustan, en gran parte, a lo señalado en estudios previos desarrollados internacionalmente, resultaría interesante desarrollar estudios futuros en esta línea con mayores tamaños muestrales y abarcando otras provincias españolas. Otra limitación derivada del número de profesorado participante en este estudio, está ligada al análisis de la existencia de posibles diferencias en la práctica de enseñanza de la composición textual implementada por el profesorado en función de su nivel de preparación, de su eficacia personal y su actitud en relación a la enseñanza de la escritura. Por ello, sería interesante explorar esta relación en futuros estudios que abarquen un mayor tamaño muestral, ya que existen evidencias empíricas que demuestran la influencia de estas variables en la práctica instruccional implementada (Sánchez-Rivero et al., 2021), siendo necesaria, además, su consideración a la hora de diseñar programas de formación del profesorado y/o de futuros docentes (Ávila-Toscano et al., 2020; Sánchez-Rivero et al., 2021). A su vez, también sería interesante, en futuros estudios, tomar medidas del rendimiento del alumnado en tareas de composición escrita, no sólo a nivel de producto textual, sino también, del proceso seguido y del tipo de estrategias cognitivas activadas por el alumnado en la escritura del texto mediante métodos on-line, como los protocolos de pensamiento en voz alta (Esteban-García et al., 2020; López et al., 2019; Saiz-Manzanares y Queiruga-Dios, 2018). En este sentido, estudios previos han mostrado que el tipo de estrategias cognitivas empleadas por el alumnado en su proceso de aprendizaje predice su rendimiento académico permitiendo diferenciar entre estudiantes con alto o bajo rendimiento (Rodríguez et al., 2017; Valiente-Barroso et al., 2020).

A pesar de las limitaciones descritas, el estudio que se presenta aborda, por primera vez, el análisis en profundidad del proceso seguido por el profesorado de $4^{\circ}$ de Educación Primaria para enseñar al alumnado la composición textual, como un primer paso en la identificación de lagunas y posibilidades de mejora en su práctica instruccional. Todo ello con el fin de ajustar el diseño de futuros programas de formación a la realidad práctica del profesorado, favoreciendo finalmente la transferencia del conocimiento científico a su práctica educativa. 


\section{- Conflicto de intereses}

Los autores declaran no tener ningún conflicto de interés.

\section{- Agradecimientos}

Durante el desarrollo de esta investigación se recibieron ayudas del Ministerio de Economía y Competitividad EDU2015-67484-P (MINECO/FEDER), proyecto de investigación concedido a la tercera autora, y del Ministerio de Educación, Cultura y Deporte (FPU15/03017), beca predoctoral concedida a la primera autora.

\section{REFERENCIAS}

Alexopoulou, A. (2010). Tipología textual y comprensión lectora en E/LE. Revista Nebrija de Lingüística Aplicada a la Enseñanza de las Lenguas, 5(9), 102113. https://revistas. nebrija.com/revistalinguistica/article/view/158

Alvarado, M., \& Silvestri, A. (2003). Introducción, la composición escrita: procesos y enseñanza. Cultura y Educación, 15(1), 7-15. https://doi. org/10.1174/113564003765202357

Arrimada, M., Torrance, M., \& Fidalgo, R. (2018). Supporting first-grade writers who fail to learn: multiple single-case evaluation of a Response to Intervention approach. Reading and Writing, 37(4), 865-891. https://doi.org/10.1007/s $11145-018$ 9817-x

Arrimada, M., Torrance, M., \& Fidalgo, R. (2019). Effects of teaching planning strategies to first-grade writers. British Journal of Educational Psychology, 89(4), 670-688. https://doi.org/10.1111/ biep.12251 doi:10.1111/biep.12251

Ávila-Toscano, J. H., Rojas-Sandoval, J., \& Tovar-Ortega, T. (2020). Perfil del dominio afectivo en futuros maestros de matemáticas. Revista de Psicología y Educación, 15(2), 225-236. https://doi. org/10.23923/rpye2020.02.197

Braaksma, M.A.H., Rijlaarsdam, G., \& van den Bergh, H. (2002). Observational learning and the effects of model-observer similarity. Journal of Educational Psychology, 94(2), 405-415. https://doi.org/10.1037/00220663.94 .2 .405

Berninger, V.W., \&Winn, W. (2006). Implications of advancements in brain research and technology for writing development, writing instruction, and educational evolution. En
C.A. MacArthur, S. Graham \& J. Fitzgerald (Eds.), Handbook of writing research (pp. 96-1 14). New York, Guildford Press.

Brindle, M., Graham, S., Harris, K.R., \& Hebert, M. (2016). Third and fourth grade teacher's classroom practices in writing: A national survey. Reading and Writing, 29(5), 929-954. https://doi.org/10.1007/ s1 $1145-015-9604-x$

Ciullo, S., Lembke, E.S., Carlisle, A., Thomas, C.N., Goodwin, M., \& Judd, L. (2016). Implementation of evidence-based literacy practices in middle school response to intervention: An observation study. Learning Disability Quarterly, 39(1), 44-57. https:// doi.org/10.1177/0731948714566120

Coker, D.L., Farley-Ripple, E., Jackson, A.F., Wen, H., MacArthur, C.A., \& Jennings, A. S. (2016). Writing instruction in first grade: An observational study. Reading and Writing, 29(5), 793-832. https://doi. org/10.1007/s $11145-015-9596-6$

Crespo, P., Jiménez, J. E., Rodríguez, C., \& González, D. (2013). El modelo de respuesta a la intervención en la Comunidad Autónoma de Canarias: nivel 2 de intervención. Revista de Psicología y Educación, 8(2), 187-203. http://www. revistadepsicologiayeducacion.es/pdf/98. pdf

De La Paz, S., \& Graham, S. (2002). Explicitly teaching strategies, skills, and knowledge: Writing instruction in middle school classrooms. Journal of Educational Psychology, 94(4), 687-698. https://doi. $\mathrm{org} / 10.1037 / / 0022-0663.94 .4 .687$

De Smedt, F., Graham, S., \& Van Keer, H. (2020). "It takes two": The added value of structured peer-assisted writing in explicit writing instruction. Contemporary Educational Psychology, 60, 101835. https://doi.org/10.1016/i. cedpsych.2019.101835

De Smedt, F., \& Van Keer, H. (2014). A research synthesis on effective writing instruction in primary education. ProcediaSocial and Behavioral Sciences, 112, 693-701. https://doi.org/10.1016/i. sbspro.2014.01.1219

Esteban-García, M., Cerezo-Menéndez, R., Cervero-Fernández, A., TueroHerrero, E., \& Bernardo-Gutiérrez, A. 
(2020). MetaTutor: revisión sistemática de una herramienta para la evaluación e intervención en autorregulación del aprendizaje. Revista de Psicología y Educación, 15(2), 108-127. https://doi. org/10.23923/rpye2020.02.191

Fidalgo, R., Torrance, M., Rijlaarsdam, G., van den Bergh, H., \& Álvarez, $M^{a}$.L. (2015). Strategy-focused writing instruction: Just observing and reflecting on a model benefits 6th grade students. Contemporary Educational Psychology, 41, 37-50. https:// doi.org/10.1016/i.cedpsych.2014.11.004

Fidalgo, R., Torrance, M., \& Robledo, P. (2011). Comparación de dos programas deinstrucción estratégica y auto-regulada para la mejora de la competencia escrita. Psicothema, 23, 672-680. https://www. redalyc.org/pdf/727/72722232023.pdf

García, J.N., \& Fidalgo, R. (2003). Diferencias en la conciencia de los procesos psicológicos de la escritura: mecánicos frente a sustantivos y otros. Psicothema, 15(1), 41-48. http://www.unioviedo.net/ reunido/index.php/PST/article/view/8135

Gottheil, B., Brenlla, M. E., Barreyro, J. P., Pueyrredón, D., Aldrey, A., Buonsanti, L., Freire, L., Rossi, A.I., \& Molina, S. (2019). Eficacia del programa "Lee Comprensivamente" para la enseñanza de estrategias de comprensión lectora en estudiantes de Educación Primaria. Revista de Psicología y Educación, 14(2), 99-111. https://doi.org/10.23923/ rpye2019.01.175

Graham, S., Berninger, V., \& Fan, W. (2007). The structural relationship between writing attitude and writing achievement in first and third grade students. Contemporary Educational Psychology, 32(3), 516536. $\quad$ https://doi.org/10.1016/i. cedpsych.2007.01.002

Graham, S., Bollinger, A., Booth, C., D'Aoust, C., MacArthur, C., McCutchen, D., \& Olinghouse, N. (2012). Teaching elementary school students to be effective writers: $A$ practice guide. Washington, DC: National Center for Education Evaluation and Regional Assistance, Institute of Education Sciences, Department of Education. http:// ies.ed.gov/ncee/wwc/publications reviews. aspx\#pubsearch
Graham, S., \& Harris, K.R. (2018). Evidencebased writing practices: A meta-analysis of existing meta-analysis. En R. Fidalgo, K.R. Harris \& M. Braaksma (Eds.), Design principles for teaching effective writing: theoretical and empirical grounded principles (pp. 13-37). Leiden: Brill Editions. Graham, S., Harris, K. R., Fink, B., \& MacArthur, C. A. (2001). Teacher efficacy in writing: A construct validation with primary grade teachers. Scientific Studies of Reading, 5(2), 177-202. https://doi. org/10.1207/S1532799Xssr0502 3

Gutiérrez, R. (2013). Actitudes hacia la escritura en alumnos sordos y oyentes integrados en contextos escolares bilingües. Profesorado. Revista de Currículum y Formación del Profesorado, 17(1), 385-400. http://www. ugr.es/ recfpro/rev171 COL8.pdf

Hayes, J. R. (1996). A new framework for understanding cognition and affect in writing. En C. Levy \& S. Ransdell (Eds.), The science of writing: Theories, methods, individual differences and applciations (pp. 1-27). Mahwah, N.J.: Lawerence Erlbaum Associates.

Hayes, J.R., \& Flower, L. (1980). Identifying the organization of writing processes. En L. Gregg \& E. Steinberg (Eds.), Cognitive processes in writing: an interdisciplinary approach (pp. 3-30). Hillsdale, NJ: Lawrence Erlbaum Associates.

Koster, M., Tribushinina, E., de Jong, P.F., \& van den Bergh, H. (2015). Teaching children to write: A meta-analysis of writing intervention research. Journal of Writing Research, 7(2), 249-274. https://doi.org/10.17239/jowr2015.07.02.2

López, P., Torrance, M., \& Fidalgo, R. (2019). La distribución temporal de los procesos de escritura y su contribución a la calidad textual al final de la educación primaria. Psicothema, 37(3), 311-318. https://doi. org/10.7334/psicothema2018.326

Loureda, O. (2009). Introducción a la tipología textual ( $2^{a}$ ed.). Madrid: Arco/Libros.

Ministerio de Educación. (2010). Evaluación general de diagnóstico de 2009. Educación primaria. Cuarto curso. Informederesultados. https://www.educacionyfp.gob.es/inee/ evaluaciones-nacionales/publicacionesanteriores/evaluaciones-2009.html 
Ministerio de Educación. (2011). Evaluación general de diagnóstico de 2010. Educación secundaria obligatoria. Segundo curso. Informe de resultados. https://www.educacionyfp.gob.es/inee/ evaluaciones-nacionales/publicacionesanteriores/evaluaciones-2010.html

National Center for Education Statistics. (2003). The nation's report card: Writing 2002. https:// nces.ed.gov/nationsreportcard/pdf/ main2002/2003529.pdf

National Center for Education Statistics. (2012). The nation's report card: Writing 2011. https:// nces.ed.gov/nationsreportcard/pdf/ main2011/2012470.pdf

Panadero, E., \& Alonso-Tapia, J. (2014). Teorías de autorregulación educativa: una comparación y reflexión teórica. Psicología Educativa, 20(1), 11-22. https://doi. org/10.1016/i.pse.2014.05.002

Rietdiik, S., van Weijen, D., Janssen, T., van den Bergh, H., \& Rijlaarsdam, G. (2018). Teaching writing in primary education: Classroom practice, time, teachers' beliefs and skills. Journal of Educational Psychology, 110(5), 640-663. https:// doi.org/10.1037/edu0000237

Robledo, P., Arias-Gundín, O., Palomo, M. P., Andina, E., \& Rodríguez, C. (2018). Perfil escritor y conocimiento metacognitivo de las tareas aca $\neg$ démicas en los estudiantes universitarios. Publicaciones, 48(1), 197-217. https://doi.org/10.30827/ publicaciones.v48i1.7335

Robledo, P., Fidalgo, R., Álvarez, M.L., \& Sánchez, R. (2016). Adquisición de estrategias de autorregulación de la escritura a través del modelado y la emulación por pares vs. individual. En J.L. Castejón (Coord.), Psicología y Educación: presente y futuro (pp. 555562). ACIPE- Asociación Científica de Psicología y Educación.

Rodríguez, S., Piñeiro, I., Regueiro, B., Estevez I., \& Val, C. (2017). Estrategias cognitivas, etapa educativa y rendimiento académico. Revista de Psicología y
Educación, 12(1), 19-34. http://www. revistadepsicologiayeducacion.es/ $\mathrm{pdf} / 143 . \mathrm{pdf}$

Saiz-Manzanares, M.C., \& Queiruga-Dios, M.A. (2018). Evaluación de estrategias metacognitivas: aplicación de métodos online. Revista de Psicología y Educación, 13(1), 33-45. https://doi.org/10.23923/ rpye2018.01.156

Sánchez-Rivero, R., Alves, R.A., Limpo, T., \& Fidalgo, R. (2021). Un estudio de encuesta sobre la enseñanza de la escritura en la educación obligatoria: prácticas y variables del profesorado. Revista Española de Pedagogía, 79(279), 321 -340. https://doi.org/10.22550/ REP79-2-2021-01

Sánchez-Rivero, R., \& Fidalgo, R. (2020). La enseñanza de la conciencia fonológica en la educación infantil: un estudio observacional. Revista de Psicología y Educación, 15(2), 184-200. https://doi. org/10.23923/rpye2020.02.195

Sánchez, G., \& Galindo, P. (2018). Uso e integración de las TIC en el aula y dificultades del profesorado en activo de cara a su integración. Profesorado. Revista de Currículum y Formación del Profesorado, 22(3), 341 -358. https://doi. org/10.30827/profesorado.v22i3.8005

Torrance, M., Fidalgo, R., \& García, J.N. (2007). The teach ability and effectiveness of cognitive self-regulation in sixth-grade writers. Learning and Instruction, 17, 265- 285. https://doi.org/10.1016/i. learninstruc. 2007.02.003

Valiente-Barroso, C., Martínez-Vicente, M., Cabal-García, P., \& AlvaradoIzquierdo, J. M. (2020). Estrés infantil, estrategias de aprendizaje y motivación académica: un modelo estructural predictor del rendimiento académico. Revista de Psicología y Educación, 15(1), 46-66.https://doi.org/10.23923/ rpye2020.01.185

VERBI Software. (2019). MAXQDA 2020 [computer software]. Berlín, Alemania: VERBI Software. Disponible en maxqda. com 